\title{
Estudo Prospectivo do Grafeno Aplicado a Polímeros em Patentes
}

\author{
Prospective Study of Graphene Applied to Polymers in Patents
}

\author{
Cintia Maria Carneiro Franco Lima ${ }^{1}$ \\ Guilherme da Mata Quintella ${ }^{1}$ \\ Lílian Maria Tosta Simplício Rodrigues ${ }^{1}$ \\ ${ }^{1}$ Universidade Federal da Bahia, Salvador, BA, Brasil
}

\begin{abstract}
Resumo
O grafeno, alótropo do carbono, é um material estratégico que apresenta uma diversidade de aplicações tecnológicas por causa das suas propriedades. A pesquisa das famílias de patentes foi realizada no banco internacional de dados europeu com o auxílio do software Orbit, versão 2019, na classificação internacional C08F. A pesquisa foi realizada a partir dos dados de domínio tecnológico, de produção anual de patentes, de P\&D e dos mercados de aplicação de polímeros em grafeno, os players, e, por fim, foi realizada uma análise do status legal dessas patentes. Essa análise permitiu identificar tendências de contínuo desenvolvimento nessa tecnologia e constatar que os maiores players e investidores atuam nos mercados chineses.
\end{abstract}

Palavras-chave: Grafeno. Polímeros. Patentes.

\begin{abstract}
Graphene, a carbon allotrope, is a strategic material and presents a diversity of technological applications due to its properties. The study of patent families was carried out in the european international database, using the software Orbit, version 2019, by the international classification C08F. The research was based on technological domain data, annual patents, R\&D and main markets for the application of graphene polymers, main players, and finally, an analysis of the legal status of these patents. The analysis allowed to identify trends of continuous development in this technology and to verify that the biggest players and the biggest investors act in the Chinese markets.
\end{abstract}

Keywords: Graphene. Polymers. Patents.

Área Tecnológica: Prospecção Tecnológica. Grafeno. Polímeros.

\section{Introdução}

A partir do ano 2010, as pesquisas científicas relacionadas ao grafeno têm aumentado exponencialmente e recebido destaque mundial, principalmente nos campos da ciência e da engenharia (SINGH et al., 2011; LUO et al., 2012). O grafeno assim como os nanotubos de carbono (Carbon Nanotubes - CNT) são considerados materiais estratégicos de diferentes aplicações tecnológicas em razão das suas propriedades (LIU et al., 2011; ZARBIN; OLIVEIRA, 2013). Além de possuírem alta resistência química, resistência à oxidação e à temperatura $e$ à baixa densidade, eles apresentam resistência mecânica devido à ligação C-C em estruturas grafíticas, flexibilidade e resistência à ruptura quando dobrados ou torcidos (LI et al., 1996). 
Tal conjunto de propriedades contribui para que esse material seja utilizado em revestimento de polímeros (OTHMAN et al., 2019; MONETTA et al., 2015), estocagem de energia (CHAVAN et al., 2019; YUE et al., 2019), eletrônica (JAFARI et al., 2014), catálise (FERREIRA; RANGEL, 2009) e nas aplicações biomédicas (YANG et al., 2013; CANCINO et al., 2014). O grafeno é uma matéria-prima cara. Ao consultar o site da empresa Sigma-Aldrich (maio/2019), verificou-se que o custo médio de apenas $500 \mathrm{mg}$ dessa substância custava em torno de $\mathrm{R} \$ 1.887,00$. Esse alto valor agregado se deve, principalmente, à complexidade nos métodos de obtenção dessa substância.

O grafeno é um alótropo do carbono e representante da família dos nanomateriais. Consiste em um arranjo bidimensional com a espessura monoatômica, formado por hexágonos de átomos de carbono com hibridização $\mathrm{sp}^{2}$, cujo empilhamento origina a estrutura do grafite, como pode ser visto na Figura 1 (TERRONES, 2003; FERREIRA; RANGEL, 2009; ZARBIN; OLIVEIRA, 2013). Esse material foi isolado e caracterizado pela primeira vez em 2004 a partir de sucessivas etapas de descasque de um pedaço de grafite com o auxílio de uma fita adesiva (NOVOSELOV et al., 2004). Esse trabalho conferiu, posteriormente, o Nobel de Física aos seus inventores no ano de 2010 (NOBEL PRIZE, 2019a). A descoberta possibilitou uma ampla variedade de aplicações práticas, por exemplo, a criação de novos materiais e a fabricação de eletrônicos inovadores. O grafeno é adequado para a produção de telas transparentes sensíveis ao toque, painéis de luz, células solares, transistores e condutores de eletricidade mais resistentes ao calor e mecanicamente mais robustos (NOBEL PRIZE, 2019b).

Figura 1 - Estrutura do grafeno

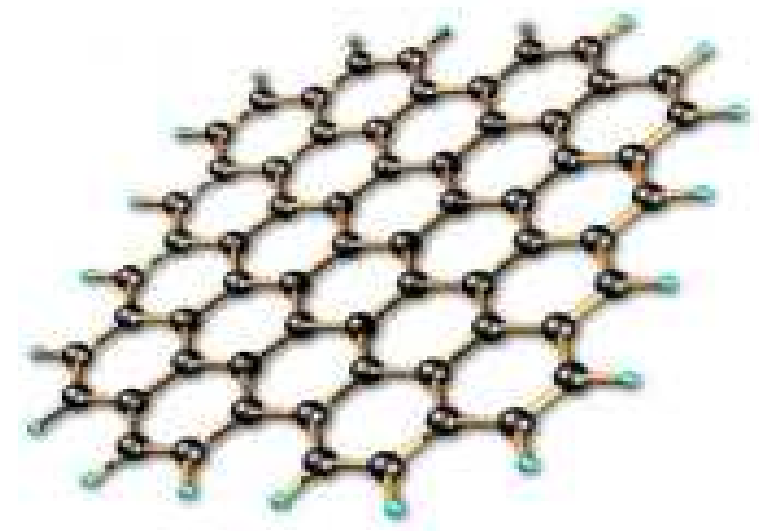

Fonte: Adaptada de Zarbin e Oliveira (2013)

A maior possibilidade de aplicação do grafeno está nos materiais compósitos (formados pela combinação de dois ou mais diferentes materiais), principalmente com os polímeros (SINGH et al., 2011). Desde a incorporação do grafeno com uma segunda fase ativa, a exemplo dos nanotubos de carbono, conduzindo a formação de polímeros e óxidos metálicos, houve uma melhora considerável no desempenho e nas propriedades físicas desses materiais. Compósitos à base de grafeno são de interesse científico e industrial e podem se tornar materiais competitivos para aplicações relacionadas à energia (LUO et al., 2012) e para o desenvolvimento de revestimentos multifuncionais (OTHMAN et al., 2019). Os nanocompósitos poliméricos à base de grafeno são preparados por meio da mistura física ou química de nano plaquetas de grafeno $e$ seus derivados com polímero (OTHMAN et al., 2019). Os nanofillers servem como uma barreira 
física que reduz a permeabilidade do revestimento do polímero por meio do alongamento do caminho no deslocamento de íons e moléculas (SAZOU; DESHPANDE, 2017; SENG et al., 2016)

Este trabalho tem como objetivo principal utilizar o mapeamento patentário por meio do software Orbit (2019) para verificar as perspectivas e as tendências no desenvolvimento da tecnologia do grafeno aplicado a polímeros. Além disso, pretende-se, ainda, identificar os principais locais de pesquisa e comercialização, apresentar os maiores apropriadores de patentes neste mercado e traçar tendências atuais e futuras.

\section{Metodologia}

A pesquisa nos bancos de patentes ocorreu no período de 31 de maio de 2019 a 17 de junho de 2019 e foi realizada nos campos de conceitos, reivindicações, objeto da invenção e reivindicações independentes do software Orbit Intelligence (ORBIT, 2019). As palavras-chaves utilizadas foram "graphene" e "polymer" com a seguinte combinação de expressões:

(graphen + AND polymer + )

Foram obtidos, preliminarmente, um total de 46.381 resultados pelo software Orbit Intelligence em 17 de junho de 2019. Esse resultado inicial foi filtrado segundo a Classificação Internacional de Patentes (IPC, 2019), utilizando-se os seguintes códigos:

a) C08 - Compostos macromoleculares orgânicos; sua preparação ou seu processamento químico; composição baseada neles.

b) $\mathrm{C} 08 \mathrm{~F}$ - Compostos macromoleculares obtidos por reações compreendendo apenas ligações insaturadas C-C.

Após, obteve-se um escopo final com 1.665 patentes que foram prospectadas e mapeadas neste artigo.

A Tabela 1 a seguir apresenta o escopo do estudo com as palavras-chave utilizadas e com os resultados obtidos.

Tabela 1 - Escopo do estudo prospectivo com os termos utilizados na busca e o número de resultados obtidos (acesso em 17/06/2019)

\begin{tabular}{cccc} 
Graphene & Polymer & C08F & Resultados \\
X & - & - & 123.700 \\
- & X & - & 3.377 .479 \\
X & X & - & 46.381 \\
X & - & $X$ & 1.838 \\
X & X & X & 1.665 \\
\hline
\end{tabular}

Fonte: Elaborada pelos autores deste artigo (2019)

Para a finalidade deste artigo, a palavra patente será utilizada para designar família de patentes. As famílias de patentes estão baseadas na data de prioridade do primeiro depósito. Dessa forma, o conteúdo tecnológico é o mesmo. 


\section{Resultados e Discussão}

Em relação ao domínio tecnológico do grafeno (Figura 2), observa-se que há uma grande concentração na área de Química macromolecular - em especial, polímeros (hexágono em vermelho em destaque). Esse valor representa quase $39 \%$ em relação aos demais segmentos como outras máquinas especiais $(11,2 \%)$, Química dos materiais básicos $(11,0 \%)$ e energia (5,7\%), por exemplo (ORBIT, 2019). Essa informação corrobora com o levantamento prévio dos artigos a respeito desse tema.

Figura 2 - Domínio tecnológico do grafeno

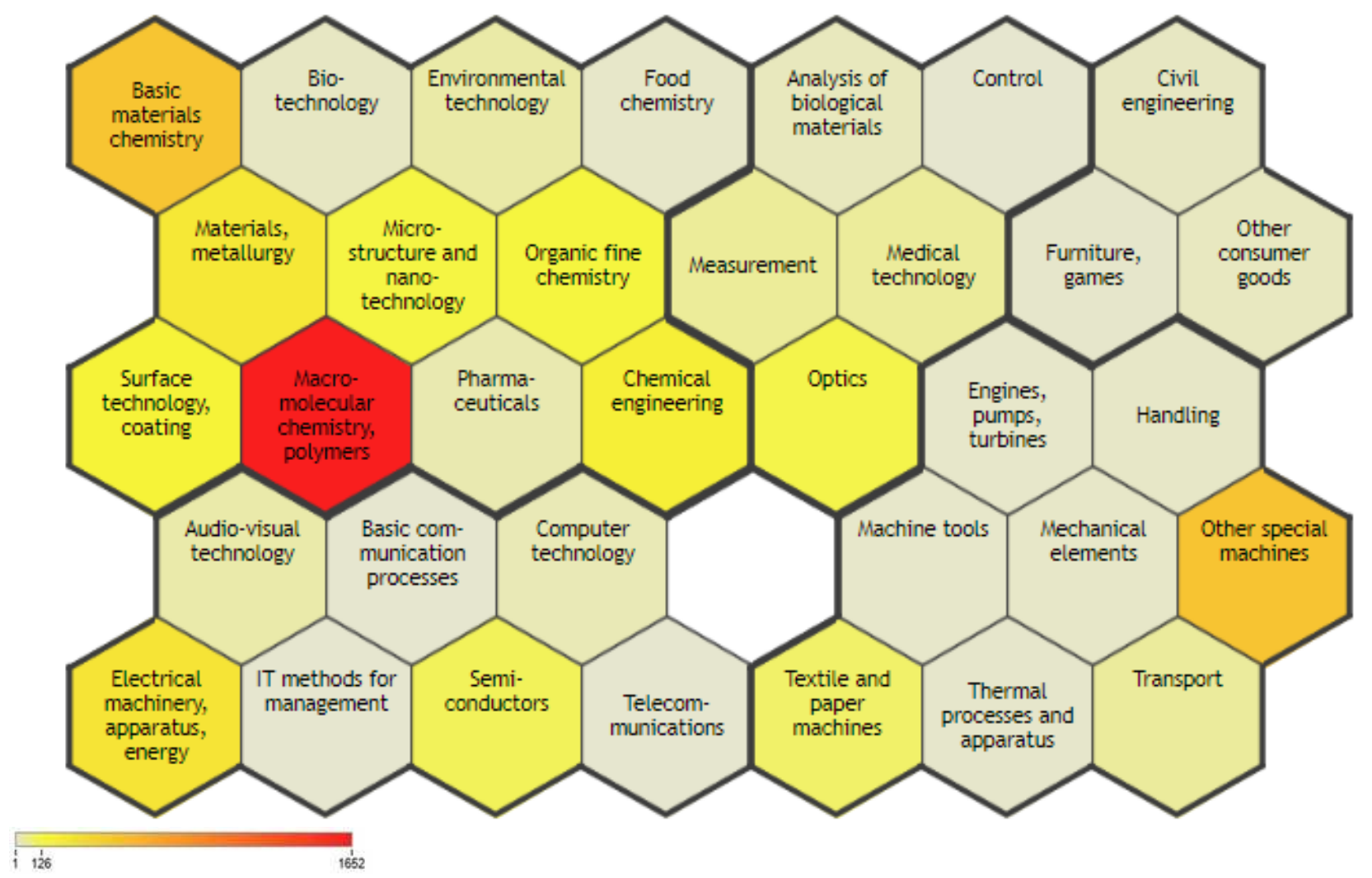

Fonte: Adaptada de Orbit (2019)

Gráfico 1 - Número total de patentes de grafeno com aplicação em polímeros submetidas por ano

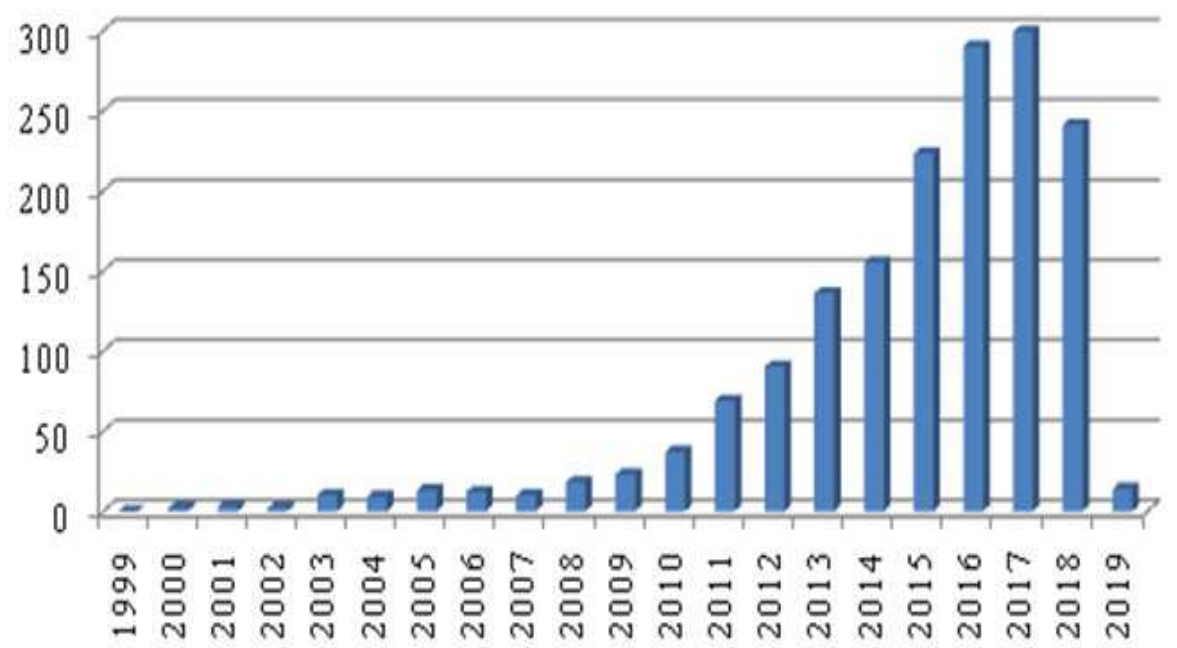

Fonte: Elaborado pelos autores deste artigo (2019) 
Verifica-se no Gráfico 1 que houve um crescimento considerável do número de patentes submetidas de grafeno com aplicação em polímeros entre o ano de 1999 até o ano de 2017, em que se registrou um máximo com um total de 299 publicações. É provável que o número de 2018 supere esse patamar, uma vez que parte das patentes protocoladas neste ano ainda está no período de sigilo e já possui um total de 290 registros.

O salto no desenvolvimento de patentes ocorreu, particularmente, após a concessão do prêmio Nobel de Física no ano de 2010, no qual os cientistas André Geim e Konstantin Novoselov demonstraram que o grafeno possuía propriedades únicas: o que gerou um grande interesse sobre o tema nas tecnologias do grafeno e também na publicidade.

A partir da Figura 3A, é possível identificar quais países possuem mais patentes por ordem de primeiro depósito, ou seja, em quais países estão acontecendo Pesquisa e Desenvolvimento (P\&D) com grafeno aplicado a polímeros. Constata-se que os principais produtores de tecnologia (P\&D) são a China (915 patentes), seguido dos Estados Unidos (279 patentes) e do Japão (207 patentes). Convém salientar o predomínio da China sobre os Estados Unidos nesse tipo de tecnologia, dada a quantidade de patentes: mais que o triplo das que foram concedidas pelos Estados Unidos. O Brasil, em contrapartida, encontra-se atualmente na $16^{\text {a }}$ posição no ranking da produção patentária investigada com apenas duas famílias de patentes (ORBIT, 2019).

A Figura 3B, à direita, identifica os principais mercados e em quais países há mais depósitos de famílias de patentes. Verifica-se que os maiores mercados potenciais são de fato os dos países que mais investem em P\&D: China, Estados Unidos e Japão, respectivamente. O Brasil, por exemplo, encontra-se em $12^{\circ}$ lugar no número de patentes de grafeno aplicadas a polímeros, com um total de 51 famílias.

Figura 3 - Mapas mundiais de patentes para grafeno com aplicação em polímeros: A) P\&D e B) principais mercados

A)

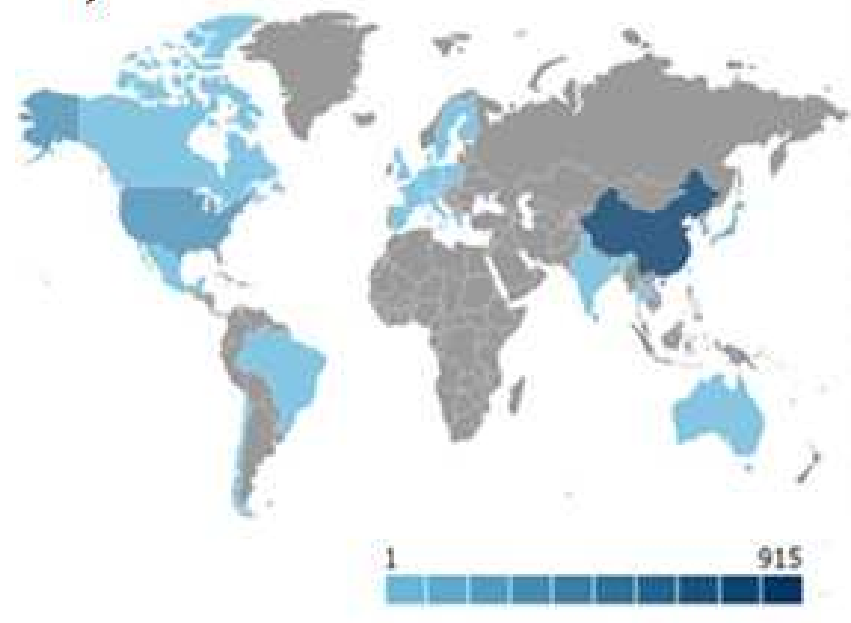

B)

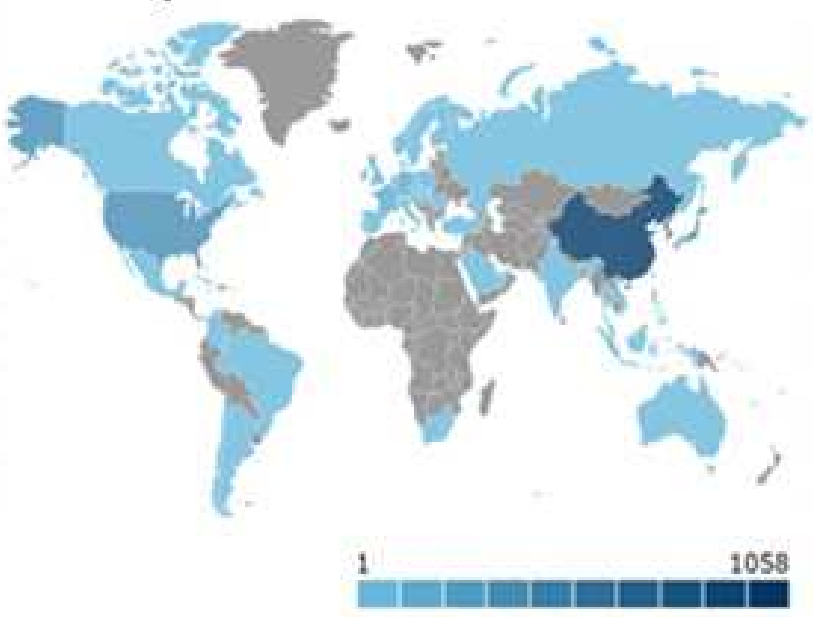

Fonte: Adaptada de Orbit (2019)

O Gráfico 2 a seguir apresenta os cinco maiores players por ordem de patentes concedidas de polímeros de grafeno. A empresa líder é a Arkema, empresa multinacional sediada na cidade de Colombes, na França, e possui a propriedade de 33 patentes. Essa multinacional foi o resultado da reestruturação da antiga Total, empresa do ramo do petróleo. Nessa reestruturação, a 
Arkema ficou com os campos de polímeros de revestimento, químicos industriais e produtos para performance. A Arkema obteve em 2017 uma receita 8,8 milhões de euros (ARKEMA 2018).

Gráfico 2 - As cinco principais empresas/centros de pesquisas no cenário internacional que investem na tecnologia do grafeno aplicado a polímeros

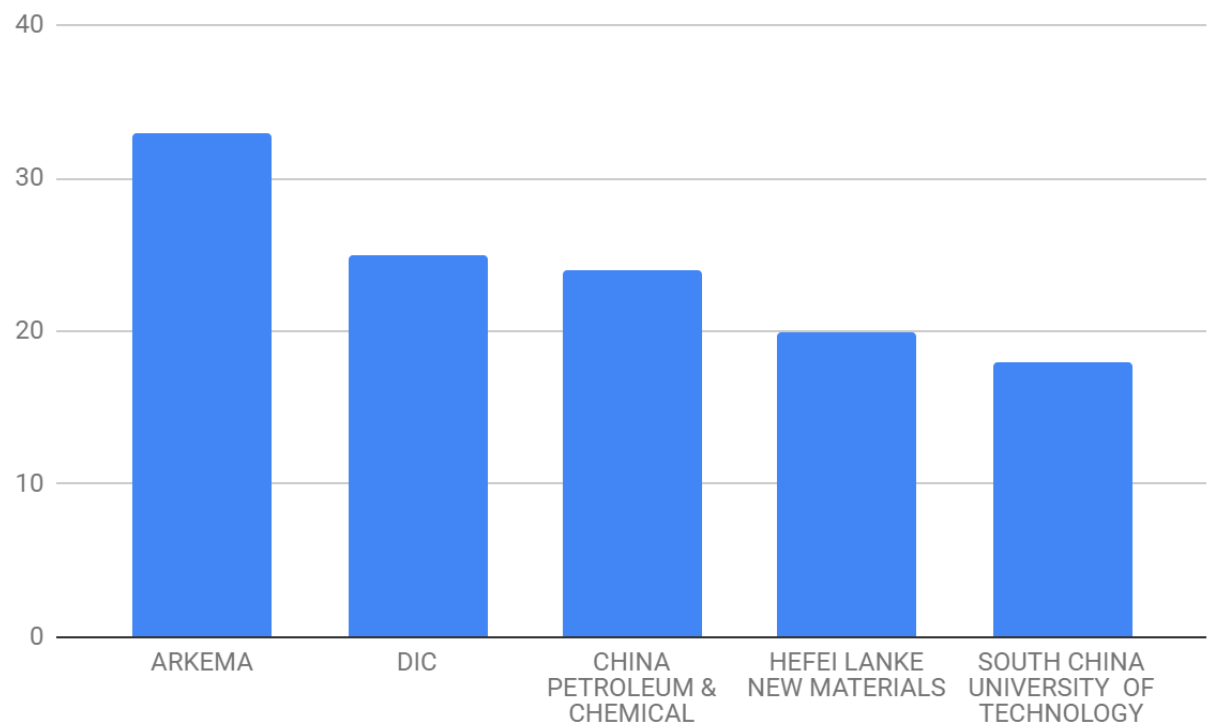

Fonte: Elaborado pelos autores deste artigo (2019)

Em segundo lugar se encontra a Dainippon Inkand Chemicals (DIC), que detém um total de 25 patentes de polímeros de grafeno. Essa empresa é uma corporação japonesa que trabalha nas áreas de tintas, pigmentos, polímeros, plásticos e componentes bioquímicos e obteve em 2017 uma renda de 789,4 bilhões de ienes (DIC 2018).

Em terceiro lugar com 24 patentes, há a China Petroleum \& Chemical Corporation, empresa multinacional chinesa cujo principal foco é a extração, refino, estoque e revenda de petróleo $e$ seus derivados. Junto com sua controladora Sinopec, ela forma o maior conglomerado de petróleo do mundo e obteve uma renda de 314,4 bilhões de dólares em 2017 (SINOPEC, 2018).

Em quarto lugar encontra-se a Hefei Lanke New Materials, uma empresa de capital fechado, situada na província de Hefei, na China, que está voltada para o desenvolvimento de novos materiais e polímeros. Como se trata de uma empresa que não tem ações comercializadas na bolsa, não é possível aferir sua renda anual. Contudo, ela mantém um total bastante expressivo de 20 patentes de polímeros de grafeno.

Situada em quinto lugar está a South China University of Technology, que é uma universidade chinesa com foco em tecnologia, localizada na província de Ghuangzhou. Essa instituição se destaca pelos cursos de engenharia e de química e detém 18 patentes.

O estudo dos players confirma as informações colhidas no concernente à pesquisa $e$ ao desenvolvimento das tecnologias de grafeno, pois há um claro predomínio de empresas e de instituições chinesas explorando essa tecnologia. Também há um evidente predomínio do uso do grafeno como revestimento a fim de obter uma linha de produção mais eficiente. O que, por sua vez, confere com o levantamento dos domínios tecnológicos e com os potenciais levantados por André Geim e Konstantin Novoselov em 2004. 
Gráfico 3 - Status Legal (em porcentagem) das patentes de grafeno com aplicação em polímeros

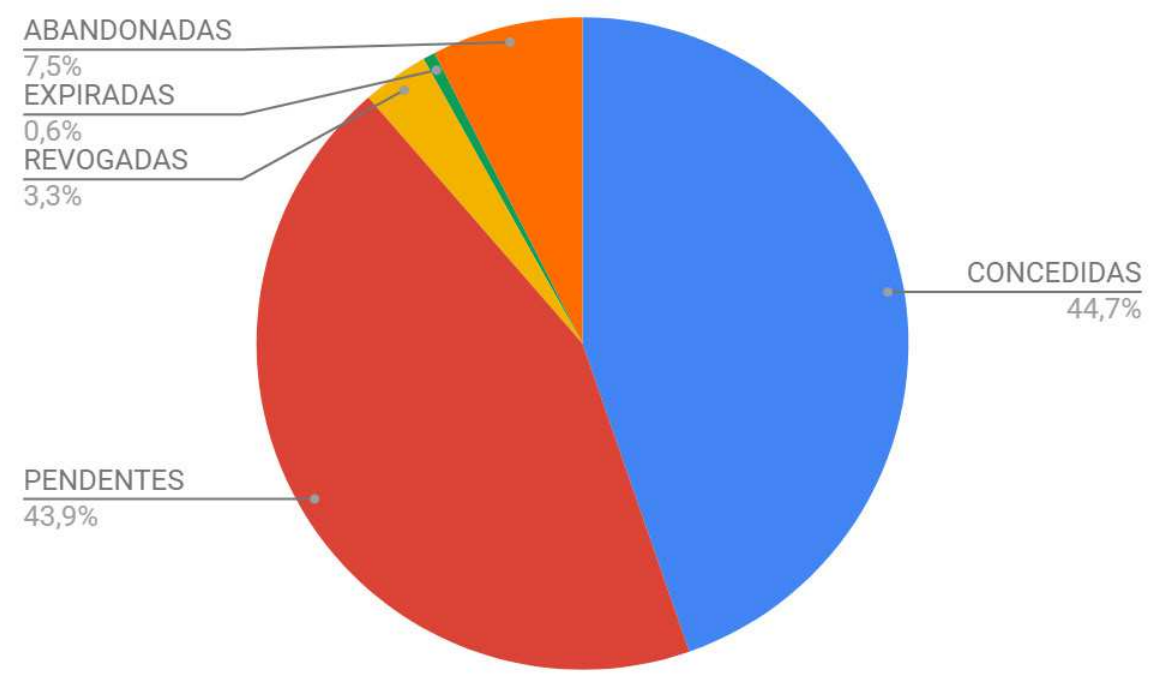

Fonte: Elaborado pelos autores deste artigo (2019)

Quanto ao status legal das patentes de grafeno com aplicação em polímeros, há 190 patentes correspondentes às patentes mortas (revogadas, expiradas e abandonadas) e 1.475 patentes relacionadas às ativas (concedidas ou pendentes). A análise do Gráfico 3 revela que a indústria de desenvolvimento tecnológico do grafeno é principiante, pois constam apenas 10 patentes expiradas até hoje. Por outro lado, é possível afirmar que esse mercado está em franca expansão, pois o número de patentes em análise (731) é quase equivalente ao número de patentes já concedidas (744). Além disso, é possível constatar que a competitividade entre os proprietários não é muito grande, já que o número de patentes revogadas é muito pequeno, havendo apenas 55 patentes em todo o mundo que foram revogadas por causa de disputas administrativas e legais.

\section{Considerações Finais}

A partir do número crescente de patentes sobre o assunto grafeno, é possível afirmar que a área de Química dos nanomateriais, em especial a de polímeros, está longe de ter estagnação. O grafeno é um material que apresenta grandes oportunidades para uma nova geração de materiais com diferentes aplicações poliméricas como pode ser verificado nos dados da Figura 2 deste artigo.

Constata-se que há uma grande concentração de mercado de grafeno na China devido à presença de diversas empresas chinesas do setor, além de ser um país que investe fortemente nesse tipo de tecnologia, tendo recebido destaque mundial.

Quanto às perspectivas futuras, não foram encontrados dados que apontem para uma diminuição no ritmo de desenvolvimento dessa tecnologia. 


\section{Referências}

ARKEMA. 2018. Disponível em: http://www.arkema.com/en/investor-relations/financials/annualreports/. Acesso em: 28 jun. 2019.

CANCINO, J. et al. Nanotecnologia em medicina: aspectos fundamentais e principais preocupações.

Química Nova, [S.l.], v. 37, n. 3, p. 521-526, 2014.

CHAVAN, S. et al. Characterization of linear low-density polyethylene with graphene as thermal energy storage material. Materials Research Express, [S.I.], v. 6, p. 1-9, 2019.

DIC. 2018.Disponível em: http://www.dic-global.com/en/csr/pdf/dic_report_en_2018_detail.pdf. Acesso em: 28 jun. 2019.

FERREIRA, H. S.; RANGEL, M. C. Nanotecnologia: aspectos gerais e potencial de aplicação em catálise. Química Nova, [S.l.], v. 32, n. 7, p. 1.860-1.870, 2009.

IPC. 2019. Disponível em: http://ipc.inpi.gov.br. Acesso em: 31 maio 2019.

JAFARI, M. et al. Effect of boron and nitrogen doping on electro-optical properties of armchair and zigzag graphyne nanoribbons. Computacional Materials Science, [S.l.], v. 82, p. 391-398, 2014.

LI, W. Z. et al. Large-Scale Synthesis of Aligned Carbon Nanotubes. Science, [S.l.], v. 274, p. 1.7011.703, 1996.

LIU, Z. et al. Carbon materials for drug delivery \& cancer therapy. Materials Today, [S.l.], v. 14, n. 7-8, p. 316-323, 2011.

LUO, B. et al. Chemical approaches towards graphene-based nanomaterials and their applications in energy-related areas. Small, [S.l.], v. 8, n. 5, p. 630-646, 2012.

MONETTA, T. Graphene/Epoxy coating as multifunctional material for aircraft structures. Aerospace, [S.I.], v. 2, p. 423-434, 2015.

NOBEL PRIZE. 2019a. Disponível em: http://www.kva.se/en/pressrum/pressmeddelanden/ nobelpriset-i-fysik-2010. Acesso em: 4 jun. 2019.

NOBEL PRIZE. 2019b. Disponível em: http://www.nobelprize.org/prizes/physics/2010/press-release/. Acesso em: 25 jun. 2019.

NOVOSELOV, K. S. et al. Electric field effect in atomically thin carbon films. Science, [S.l.], v. 306, p. 666-669, 2004.

ORBIT de Paris, França. Banco de dados. 2019. Disponível em: http://www.orbit.com. Acesso em: 7 jun.2019.

OTHMAN, N. H. et al. Graphene-based polymer nanocomposites as barrier coating for corrotion protection. Progress in Organic Coating, [S.l.], v. 135, p. 82-99, 2019.

SAZOU, D.; DESHPANDE, P. P. Conducting polyaniline nanocomposite-based paints for corrosion protection of steel. Chemical Papers, [S.l.], v. 71, p. 459-487, 2017.

SENG, X. et al. Synthesis of functionalized graphene/polyaniline nanocomposites with effective synergistic reinforcement on anticorrosion. Industrial \& Engineering Chemistry Research, [S.I.], v. 55 , p. 8.576-8.585, 2016. 
SIGMA-ALDRICH. 2019. Disponível em: http://www.sigmaaldrich.com. Acesso em: 31 maio 2019.

SINGH, V. et al. Graphene based materials: past, present and future. Progress in Materials

Science, [S.I.], v. 56, p. 1.178-1.271, 2011.

SINOPEC, 2018. Disponívelem: http: www.sinopec.com/listco/en/Resource/Pdf/20180325036.pdf. Acesso em: 28 jun. 2019.

TERRONES, M. Science and technology of the twenty-first century: synthesis, properties, and applications of carbon nanotubes. Annual Review of Material Research, [S.l.], v. 33, p. 419-501, 2003.

YANG, K. et al. Nano-graphene in biomedicine: theranostic applications. Chemical Society Review, [S.l.], v. 42, p. 530, 2013.

YUE, Z. et al. A novel strategy of one device achieves two functions: energu storage and temperature sense multi-functions device based on graphene planar-structure supercapacitor. Materials

Research Society Advances, [S.1.], v. 4, n. 23, p. 1.321-1.326, 2019.

ZARBIN, A. J. G. Química de (nano) materiais. Química Nova, [S.l.], v. 30, n. 6, p. 1.469-1.479, 2007.

ZARBIN, A. J. G.; OLIVEIRA, M. M. Nanoestruturas de carbono (nanotubos, grafeno): quo vadis?

Química Nova, [S.1.], v. 36, n. 10, p. 1.533-1.539, 2013.

\section{Sobre os Autores}

\section{Cintia Maria Carneiro Franco Lima}

E-mail: cintialima.quimica@gmail.com

Mestrado em Química pela Universidade Federal da Bahia em 2017.

Endereço profissional: Universidade Federal da Bahia, Instituto de Química, Rua Barão de Jeremoabo, n. 147,

Ondina, Salvador, BA. CEP: 40170-115.

\section{Guilherme da Mata Quintella}

E-mail: guilherme.quintella@gmail.com

Bacharel em Direito pela Universidade Católica do Salvador em 2013.

Endereço profissional: Quintella e Sicupira Advogados Associados, Avenida Tancredo Neves, n. 1.632, Edifício

Salvador Trade Center, Salas 505 e 506, Torre Sul, Salvador, BA. CEP: 41820-021.

\section{Lílian Maria Tosta Simplício Rodrigues}

E-mail:mlilian@ufba.br

Doutorado em Química pela Universidade Federal da Bahia em 2009.

Endereço profissional: Universidade Federal da Bahia, Instituto de Química, Rua Barão de Jeremoabo, n. 147,

Ondina, Salvador, BA. CEP: 40170-115. 